\title{
Geographical distribution and Host Range of Genus Uromyces (Pucciniaceae, Uredinales) in Pakistan
}

\author{
N.S. AFSHAN ${ }^{1}$, A. Ishaq ${ }^{2}$, A. R. Niazi ${ }^{2} \&$ A.N. Khalid ${ }^{2}$ \\ ${ }^{1}$ Centre for Undergraduate Studies, University of the Punjab, Lahore, Pakistan \\ ${ }^{2}$ Department of Botany, University of the Punjab, Lahore, Pakistan \\ Corresponding author: Dr. Najam-ul-Sehar Afshan
}

\begin{abstract}
This paper represents a checklist of rust fungi of Genus Uromyces (Link) Unger in Pakistan together with their known host plants. A total of sixty four (64) are mentioned here. This number may include two (02) indigenous species of rusts. Several specimens have been found rare, being known from a limited number of collections. Species identification, distribution and host index have been provided.
\end{abstract}

Key Words: Biodiversity, leguminous rust, Biocontrol, Host specificity

\section{Introduction}

Uromyces (Link) Unger, the second largest genus of Uredinales (Rust fungi) next to Puccinia Pers., parasitizes economically important plants throughout the world. This genus contains about 600 species distributed worldwide, among which sixty four (64) species have been reported in Pakistan till 2013, parasitizing sixteen (16) plant families. Fabaceae has been found having most potential hosts for this genus in Pakistan as thirty (30) species have been recorded on members of this family. Fourteen (14) species on Poaceous hosts, four (04) on Polygonaceae and Euphorbiaceae each, three (03) on Chenopodiaceae and one (01) species of Uromyces on Acanthaceae, Boraginaceae, Caryophyllaceae, Cyperaceae, Liliaceae, Geraniaceae, Plumbaginaceae, Ranunculaceae and Valerianaceae each, have been recorded while one (01) species is found having wide host range being a parasite of both Euphorbiaceae and Papilionaceae. Occurrence of maximum number of Uromyces species on fabaceous from Pakistan is in line with data from other countries. This manuscript is a compilation of available species of Uromyces reported from, or know to occur, in Pakistan. Species identification, distribution and host index have been provided. This data will help in further studies related to diversity of this genus and its usage as biocontrol agents against noxious poaceous weeds.

\section{Material and Method}

The checklist is based on published literature and study of herbarium specimens (LAH Herbarium). The current names of fungi are given according to www.indexfungorum.org and nomenclature of host plants are given according to www.ipni.org. 


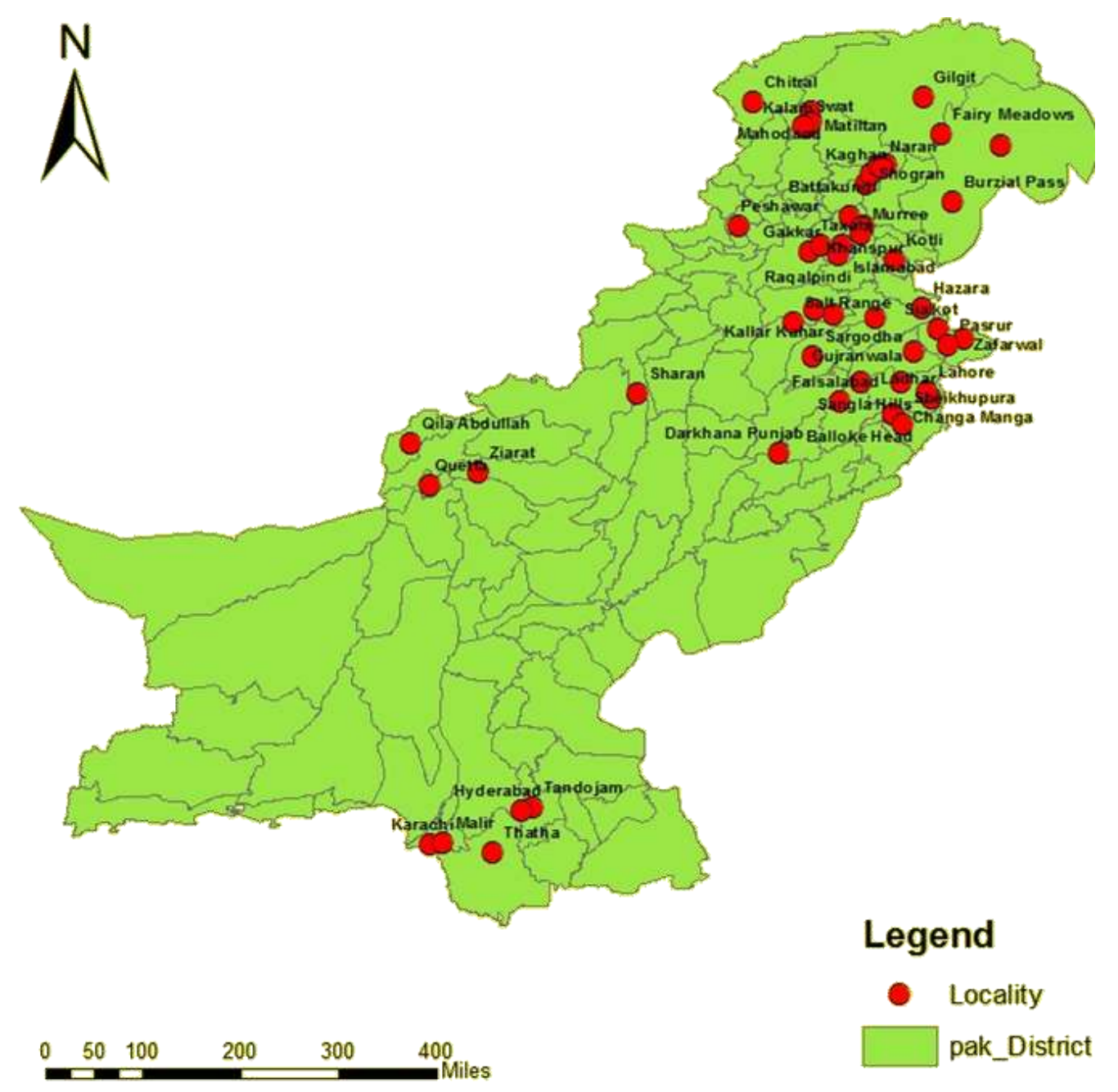

Fig. 1: Map showing distribution of Uromyces in Pakistan.

TABLE I: Table showing different species of Uromyces, their Host plants and distribution in Pakistan.

\begin{tabular}{|c|c|c|c|c|}
\hline $\begin{array}{l}\text { Sr. } \\
\text { No. }\end{array}$ & Fungus & Host Plant & Locality & Reference \\
\hline 1 & $\begin{array}{l}\text { Uromyces } \\
\text { acantholimonis Syd. \& P. } \\
\text { Syd. }\end{array}$ & $\begin{array}{l}\text { Acantholimon munroanum } \\
\text { Aitch. \& Hemsl. }\end{array}$ & Ziarat, & Ahmad, 1964, 1969 \\
\hline 2 & U. alhaginis Szemb. & Alhagi maurorum Medik. & $\begin{array}{l}\text { Balloke Head } \\
\text { Hyderabad, Lahore }\end{array}$ & $\begin{array}{l}\text { Ahmad, 1967; Ahmad, 1969; } \\
\text { Khan \& Kamal, } 1968\end{array}$ \\
\hline 3 & $\begin{array}{l}\text { U. anthyllidis (Grev.) J. } \\
\text { Schröt. }\end{array}$ & Trigonella gracilis Benth. & $\begin{array}{lr}\text { Chillan } & \text { wala, } \\
\text { Faisalabad, Gakkar, } & \text { Gaka } \\
\text { Murree, Sangla Hills }\end{array}$ & Ahmad, 1956a,b \\
\hline 4 & $\begin{array}{l}\text { U. appendiculatus (Pers.) } \\
\text { Link }\end{array}$ & $\begin{array}{l}\text { Dolichos lablab L., Lablab } \\
\text { purpureus (L.) Sweet }\end{array}$ & Kulali (Swat) & Ahmad, 1956a, b \\
\hline 5 & U. behenis (DC.) Unger & $\begin{array}{l}\text { Silene aucheriana Boiss., } S . \\
\text { vulgare (Moench) Garcke }\end{array}$ & Quetta, Sharan, Swat & $\begin{array}{l}\text { Ahmad, 1956a, b; Khalid et al., } \\
\text { 1995; Ono \& Kakishima, } 1993\end{array}$ \\
\hline 6 & U. capitatus Syd. \& P. Syd. & $\begin{array}{l}\text { Desmodium elegans Schltdl., D. } \\
\text { triflorum (L.) DC. }\end{array}$ & $\begin{array}{l}\text { Lahore, Saidan Shah, } \\
\text { Salt range, Sangla Hills, }\end{array}$ & $\begin{array}{l}\text { Ahmad, 1956a, b; Khalid et al., } \\
1993\end{array}$ \\
\hline 7 & $\begin{array}{l}\text { U. chenopodii (Duby) J. } \\
\text { Schröt. }\end{array}$ & $\begin{array}{l}\text { Suaeda fruticosa ex J.F.Gmel., } \\
\text { S. monoica ex J.F.Gmel. }\end{array}$ & Karachi & $\begin{array}{l}\text { Ghaffar \& Abbas, 1972; Khan \& } \\
\text { Kamal, } 1968\end{array}$ \\
\hline 8 & $\begin{array}{lr}U . & \text { ciceris-arietini } \\
\text { (Grognot) Jacz. \& G. Boyer }\end{array}$ & Cicer arietinum $\mathrm{L}$. & $\begin{array}{l}\text { Chillan wala, Sangla } \\
\text { Hills, Tandojam }\end{array}$ & $\begin{array}{l}\text { Ahmad, 1956a, b; Khan \& } \\
\text { Kamal, 1968 }\end{array}$ \\
\hline 9 & $\begin{array}{l}\text { U. ciceris-soongaricae } \mathrm{S} . \\
\text { Ahmad }\end{array}$ & Cicer soongaricum DC. & Swat & Ahmad, 1969 \\
\hline
\end{tabular}




\begin{tabular}{|c|c|c|c|c|}
\hline 10 & U. clignyi Pat. \& Har. & 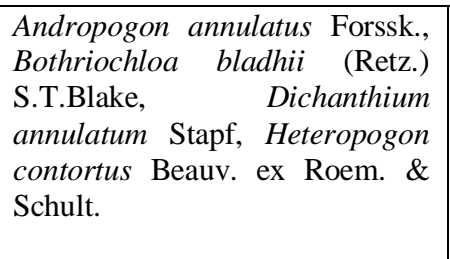 & $\begin{array}{l}\text { Chillanwala, } \\
\text { Gujranwala, Kaghan, } \\
\text { Kalam, Kallar Kahar, } \\
\text { Karachi, } \\
\text { Kulali, Rawalpindi, } \\
\text { Sangla Hills, Swat, } \\
\text { Tandojam }\end{array}$ & $\begin{array}{l}\text { Ahmad, 1956a, b; Ahmad \& } \\
\text { Arshad, 1972; } \\
\text { Ahmad, 1976; } \\
\text { Ghaffar \& Kafi, 1968; Hasnain } \\
\text { et al., 1959; Khan \& Kamal, } \\
\text { 1968; }\end{array}$ \\
\hline 11 & $\begin{array}{l}\text { U. decorates Syd. \& } \mathrm{P} . \\
\text { Syd. }\end{array}$ & $\begin{array}{l}\text { Crotalaria juncea L., Crotolaria } \\
\text { sp. }\end{array}$ & Sialkot, Zafarwal & $\begin{array}{l}\text { Ahmad, 1956a, b; Ahmad, 1964; } \\
\text { Ahmad, } 1967\end{array}$ \\
\hline 12 & $\begin{array}{l}\text { U. euphorbiae-corniculatae } \\
\text { Jordi }\end{array}$ & Lotus corniculatus L. & Swat & Khalid et al., 1995 \\
\hline 13 & U. eragrostidis Tracy & $\begin{array}{l}\text { Desmostachya bipinnata (L.) } \\
\text { Stapf }\end{array}$ & Lahore & $\begin{array}{l}\text { Ahmad \& Kamal, 1968; Ghaffar } \\
\text { \& Kafi, } 1968\end{array}$ \\
\hline 14 & U. ervi (Wallr.) Westend. & Lens culinaris Medik. & Pasrur & Ahmad, 1968 \\
\hline 15 & $\begin{array}{l}\text { U. euphorbiae Cooke \& } \\
\text { Peck }\end{array}$ & $\begin{array}{l}\text { Euphorbiae indica Lam., } \quad E . \\
\text { prostate Aiton }\end{array}$ & Lahore, Tandojam & $\begin{array}{l}\text { Ahmad, 1956a, b; Khalid et al., } \\
\text { 1995; Khan \& Kamal, 1968; } \\
\text { Ghaffar \& Kafi, 1968; Hasnain } \\
\text { et al., } 1959\end{array}$ \\
\hline 16 & U. fallens (Arthur) Barthol. & Trifolium alexandrianum $\mathrm{L}$. & Lahore & Khalid et al., 1995 \\
\hline 17 & U. flectens Lagerh. & Trifolium repens $\mathrm{L}$. & Quetta, Shoran & $\begin{array}{l}\text { Ahmad, 1956a, b; Malik \& Virk, } \\
\text { 1968; Malik et al., } 1968\end{array}$ \\
\hline 18 & U. fragilipes Tranzschel & \begin{tabular}{ll|} 
Eremopyrum & bonarpartis \\
(Spreng.) Nevski & \\
\end{tabular} & Sheikhupura & Khalid \& Iqbal, 1996 \\
\hline 19 & U. fritillariae Thüm. & Fritillaria roylei Hook. & Kaghan, Saiful Malook & Ahmad, 1956a \\
\hline 20 & $\begin{array}{l}\text { U. geranii (DC.) G.H. Otth } \\
\text { \& Wartm. }\end{array}$ & $\begin{array}{l}\text { Geranium aconitiflolium Knaf } \\
\text { ex Nyman, G. donianum Hook.f. } \\
\& \text { Thomson, G. wallichianum } \\
\text { D.Don }\end{array}$ & $\begin{array}{l}\text { Gilgit, } \\
\text { Mahodand, } \\
\text { Shogran }\end{array}$ & $\begin{array}{l}\text { Ahmad, 1956a, b; Kakishima et } \\
\text { al., 1993; Ono \& Kakishima, } \\
1992\end{array}$ \\
\hline 21 & $\begin{array}{l}\text { U. glycyrrhizae (Rabenh.) } \\
\text { Magnus }\end{array}$ & Glycerrihiza sp., G. glaba L. & Gilgit & Ahmad, 1969 \\
\hline 22 & U. heliotropii Sred. & Heliotropium sp. & Karga Nala (Gilgit) & Ahmad, 1960; 1968 \\
\hline 23 & U. heteromallus Syd. & $\begin{array}{l}\text { Haloxylon recurvum Bunge ex } \\
\text { Boiss. }\end{array}$ & Darkhana (Punjab) & Ahmad, 1956a, b \\
\hline 24 & $\begin{array}{l}\text { U. ignobilis (Syd. \& } \mathrm{P} . \\
\text { Syd.) Arthur }\end{array}$ & Sporobolus arabicus Boiss. & Lahore & Ahmad, 1956a, b \\
\hline 25 & U. inayati Syd. \& P. Syd. & Apluda mutica var. aristidae L. & Shogran & $\begin{array}{l}\text { Ahmad, 1969; Jorstad \& Iqbal, } \\
1967\end{array}$ \\
\hline 26 & U. kondoi Miura & Gueldenstaedtia sp. & Rawalpindi & Ahmad, 1956a, b \\
\hline 27 & U. laburni (DC.) G.H. Otth & Caragana ambigua Stocks & Quetta, Ziarat & Ahmad, 1956a, b \\
\hline 28 & U. lapponicus Lagerh. & $\begin{array}{l}\text { Astragalus maddenianus Benth. } \\
\text { ex Baker }\end{array}$ & $\begin{array}{ll}\text { Battakundi, } & \text { Burzial } \\
\text { Pass, Kaghan } & \end{array}$ & $\begin{array}{l}\text { Ahmad, 1956a; Cummins, 1942; } \\
\text { Ono, } 1992\end{array}$ \\
\hline 29 & $\begin{array}{lr}U . & \text { lespedezae- } \\
\text { procumbentis } & \text { (Schwein.) } \\
\text { Lagerh. } & \\
\end{array}$ & $\begin{array}{l}\text { Lespedeza sericea } \quad \text { (Thunb.) } \\
\text { Miq. }\end{array}$ & Abbottabad, Murree, & Ahmad, 1956a, b \\
\hline 30 & U. holwayi Lagerh. & Lilium sp. & $\begin{array}{l}\text { Bagh, Qila Abdullah, } \\
\text { Quetta, Shaila }\end{array}$ & Petrack, 1966 \\
\hline 31 & $\begin{array}{l}\text { U. lineolatus (Desm.) J. } \\
\text { Schröt. }\end{array}$ & Scirpus maritimus L. & 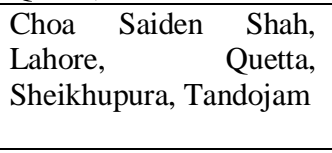 & $\begin{array}{l}\text { Ahmad, 1956a, b; Khalid et al., } \\
\text { 1993; Khan \& Kamal, 1968; ; } \\
\text { Malik \& Virk, 1968; Malik et } \\
\text { al., } 1968\end{array}$ \\
\hline 32 & $\begin{array}{l}\text { U. lycoctoni (Kalchbr.) } \\
\text { Fuckel }\end{array}$ & Aconitum sp. & Kaghan, & Ahmad, 1956a, b \\
\hline 33 & U. minor J. Schröt. & Trifolium resupinatum $\mathrm{L}$. & $\begin{array}{l}\text { Chillanwala, } \\
\text { Sheikhupura }\end{array}$ & $\begin{array}{l}\text { Ahmad, 1956a, b; Sydow and } \\
\text { Ahmad, } 1939\end{array}$ \\
\hline 34 & $\begin{array}{l}\text { U. orientalis Syd. \& } \mathrm{P} . \\
\text { Syd. }\end{array}$ & Indigofera linifolia (L.f.) Retz. & Ladhar, Zafarwal & Ahmad, 1956a, 1964a \\
\hline 35 & U. penniseti S. Ahmad & Pennisetum lanatum Klotzsch & Leepa Valley, Naran & Ahmad, 1969; Saba et al., 2011 \\
\hline 36 & $\begin{array}{ll}U . & \text { phacae-frigidae } \\
\text { (Wahlenb.) Har. }\end{array}$ & Astragalus coluteocarpus Boiss. & Barum Valley (Chitral) & Ahmad, 1956a, b \\
\hline 37 & U. pisi (Pers.) de Bary & $\begin{array}{ll}\text { Euphorbia } & \text { esula } \\
\text { Euphorbia sp. } & \end{array}$ & Chitral, Naran & $\begin{array}{l}\text { Ahmad, 1956a, b; Malik \& Virk. } \\
1968\end{array}$ \\
\hline
\end{tabular}




\begin{tabular}{|c|c|c|c|c|}
\hline 38 & $\begin{array}{ll}U . & \text { polygoni-aviculare } \\
\text { (Pers.) } & \end{array}$ & $\begin{array}{l}\text { Lathyrus sativus L., Polygonum } \\
\text { aviculare Bert. ex Meisn. P. } \\
\text { viviparum L., P. paronychioides } \\
\text { C.A.Mey. }\end{array}$ & $\begin{array}{l}\text { Hazara, Kaghan Quetta, } \\
\text { Sharan, } \\
\text { Swat }\end{array}$ & $\begin{array}{l}\text { Ahmad, 1956a, b; Malik \& } \\
\text { Khan, 1944; Malik \& Virk, } \\
\text { 1968; Malik et al., 1968; Ono, } \\
1992\end{array}$ \\
\hline 39 & U. punctatus J. Schröt. & $\begin{array}{l}\text { Oxytropis sp., } \\
\text { stracheyi } \text { Boiss. }\end{array}$ & Batakundi, Kaghan & Ahmad, 1956a, b; Ono, 1992 \\
\hline 40 & $\begin{array}{l}\text { U. rumicis (Schumach.) G. } \\
\text { Winter }\end{array}$ & Rumex chalepensis Mill. & $\begin{array}{lr}\text { Changa } & \text { Manga, } \\
\text { Chillianwala, } & \text { Choa } \\
\text { Saiden, } & \text { Lahore, } \\
\text { Peshawar, Shah, Salt } \\
\text { Range }\end{array}$ & $\begin{array}{l}\text { Ahmad, 1956a, b; Ahmad \& } \\
\text { Arshad, 1972; Khalid et al., } \\
\text { 1993; Khan \& Kamal, } 1968\end{array}$ \\
\hline 41 & U. salsolae Rabenh. & Salsola kali L. & Quetta & Ahmad, 1960, 1969 \\
\hline 42 & $\begin{array}{l}\text { U. schoenanthi Syd. \& P. } \\
\text { Syd. }\end{array}$ & Apluda mutica $\mathrm{L}$. & Lahore & Ahmad, 1956a, b \\
\hline 43 & $\begin{array}{ll}U . & \text { setariae-italicae } \\
\text { Yoshino } & \end{array}$ & $\begin{array}{l}\text { Brachiaria reptans }(\mathrm{L} .) \\
\text { C.A.Gardner \& } \text { C.E.Hubb., B. } \\
\text { ramosa Stapf, } \\
\text { geniculata }\end{array}$ & $\begin{array}{l}\text { Faisalabad, Islamabad, } \\
\text { Ladhar, Lahore, Malir, } \\
\text { Tandojam, Taxala }\end{array}$ & $\begin{array}{l}\text { Afshan et al., 2008; Ahmad, } \\
\text { 1956a, b; Ahmad, 1976; Ghaffar } \\
\text { \& Kafi, 1968; Husnain et al., } \\
\text { 1959; Khan \& Kamal, 1968; } \\
\text { Kakishima et al., 1993b }\end{array}$ \\
\hline 44 & U. sophorae Peck & $\begin{array}{l}\text { Sophora alopecuroides L., } S . \\
\text { mollis (Royle) Graham ex Bake }\end{array}$ & Gilgit & Kaneko, 1993 \\
\hline 45 & U. striatus J. Schröt. & $\begin{array}{l}\text { Argyrolobium } \text { roseum } \\
\text { (Cambess.) Jaub. \& Spach, } \\
\text { Medicago lupulina L., M. sativa } \\
\text { L., }\end{array}$ & $\begin{array}{l}\text { Balloke } r \text { Head, } \\
\text { Chillianwala, Kotli, } \\
\text { Ladhar, Malir, Quetta, } \\
\text { Raqalpindi, Sargodha, } \\
\text { Sialkot, Tandojam }\end{array}$ & $\begin{array}{l}\text { Ahmad, 1956a, b; Ahmad, 1967; } \\
\text { Ahmad, 1969; Ahmad \& } \\
\text { Arshad, 1972; Ghaffar \& Kafi, } \\
\text { 1968; Hasnain et al., 1959; Khan } \\
\text { \& Kamal, 1968; Malik \& Virk, } \\
\text { 1968; Malik et al., } 1968\end{array}$ \\
\hline 46 & U. striolatus Tranzschel & Euphorbia esula L. & Barum Valley & Ahmad, 1956a, b \\
\hline 47 & U. strobilanthis Barclay & $\begin{array}{ll}\text { Pteracanthus } & \text { urticifolius } \\
\text { (Kuntze) Bremek. } & \\
\end{array}$ & Chitral, Sharan & Ahmad, 1956a, b; Ono, 1992 \\
\hline 48 & $\begin{array}{l}\text { U. superfluus P. Syd. \& } \\
\text { Syd. }\end{array}$ & Panicum antidotale Retz. & Changa Manga, Karachi & $\begin{array}{l}\text { Ahmad, 1956a; Ghaffar \& Kafi, } \\
\text { 1968; Hasnain et al., } 1959\end{array}$ \\
\hline 49 & U. tenuicutis McAlpine & Sporobolus arabicus Boiss. & Lahore & $\begin{array}{l}\text { Ahmad, 1956a,b; Masood et al., } \\
1995\end{array}$ \\
\hline 50 & U. thellungi Maire & Rumex vesicarius $\mathrm{L}$. & Karachi, Peshawar & $\begin{array}{l}\text { Ahmad, 1956a,b; Ghaffar \& } \\
\text { Kafi, 1968; Hasnain et al., 1959; } \\
\text { Jafri, 1968; Petrak, 1966; }\end{array}$ \\
\hline 51 & U. trifolii sensu auct. & $\begin{array}{l}\text { Trifolium alaxandrianum L., } T . \\
\text { repense L., T. resupinatum L., }\end{array}$ & $\begin{array}{l}\text { Fairy Meadows, } \\
\text { Peshawar, Sangla Hills, } \\
\text { Sargodha } \\
\end{array}$ & $\begin{array}{l}\text { Afshan et al., 2012; Ahmad, } \\
\text { 1956a,b; Khan \& Kamal, 1968; } \\
\text { Malik \& Khan, 1944; }\end{array}$ \\
\hline 52 & U. trifolii-repentis Liro & Trifolium repens $\mathrm{L}$. & Gilgit, Kaghan, Quetta & $\begin{array}{l}\text { Ahmad, 1956a, b; Malik \& Virk, } \\
1968\end{array}$ \\
\hline 53 & $\begin{array}{l}\text { U. trifoli-repentis var. } \\
\text { fallens (Arthur) Cummins }\end{array}$ & Trifolium pretense $\mathrm{L}$. & Kaghan & Ono, 1992 \\
\hline 54 & U. tuberculatus Fuckel & Euphorbia sp. & Quetta & $\begin{array}{l}\text { Ahmad, 1956a, b; Malik \& Virk, } \\
\text { 1968; Malik et al., } 1968\end{array}$ \\
\hline 55 & $\begin{array}{lr}U . & \text { valerianae- } \\
\text { wallichii Arthur } & \& \\
\text { Cummins } & \\
\end{array}$ & $\begin{array}{l}\text { Valeriana jatamansi Jones ex } \\
\text { Roxb. }\end{array}$ & Changla gali, Swat & $\begin{array}{l}\text { Ahmad, 1956a, b; Ono, 1992; } \\
\text { Ono \& Kakishima, } 1992\end{array}$ \\
\hline 56 & $\begin{array}{l}\text { U. viciae-fabae var. fabae } \\
\text { (Pers.) J. Schröt. }\end{array}$ & $\begin{array}{l}\text { Lathyrus odoratus L., Pisum } \\
\text { sativum L., Vicia faba L., Vicia } \\
\text { sativa L. }\end{array}$ & $\begin{array}{l}\text { Chillianwala, } \\
\text { Faisalabad, Peshawar, } \\
\text { Quetta, Salt Range } \\
\end{array}$ & $\begin{array}{l}\text { Ahmad, 1956a, b; Malik \& Virk, } \\
1968\end{array}$ \\
\hline 57 & U. vossiae Barclay & Phacelurus speciosus C.E.Hubb. & $\begin{array}{l}\text { Fairy Meadows, } \\
\text { Kaghan, Khanspur, } \\
\text { Leepa Valley, }\end{array}$ & $\begin{array}{l}\text { Afshan et al., 2008; Ahmad, } \\
\text { 1969; Saba et al., 2011; Saba et } \\
\text { al., } 2013\end{array}$ \\
\hline 58 & U. dactylidis G.H. Otth & Agrostis gigantea Roth & Khanspur & Afshan et al., 2008 \\
\hline 59 & U. graminicola Burrill & Panicum antidotale Retz. & Khanspur & Afshan et al., 2008 \\
\hline 60 & $\begin{array}{l}\text { U. peglerae var. peglerae } \\
\text { Pole-Evans }\end{array}$ & Digitaria adscendens Henrard & Lahore & Afshan et al., 2008 \\
\hline 61 & $\begin{array}{l}\text { U. polygoni-avicularis } \\
\text { (Pers.) G.H. Otth }\end{array}$ & $\begin{array}{l}\text { Bistorta vivipara (L.) Gray, } \\
\text { Polygonum aviculare Bert. ex } \\
\text { Meisn. }\end{array}$ & $\begin{array}{l}\text { Bial Camp, Fairy } \\
\text { Meadows }\end{array}$ & Afshan et al., 2011 \\
\hline
\end{tabular}




\begin{tabular}{|r|l|l|l|l|}
\hline 62 & $\begin{array}{l}\text { U. hedysari-obscuri (DC.) } \\
\text { Carestia \& Picc. }\end{array}$ & Hedysarum falconeri Baker & Fairy Meadows & Afshan et al., 2011 \\
\hline 63 & $\begin{array}{l}\text { U. appendiculatus (Pers.) } \\
\text { Link }\end{array}$ & Vigna mungo (L.) Hepper & Leepa Valley & Afshan et al., 2011 \\
\hline Lindq. sporobolicola J.C. & $\begin{array}{l}\text { Sporobolus marginatus Hochst. } \\
\text { ex A.Rich. }\end{array}$ & Sialkot & Ishaq et al., 2011 \\
\hline
\end{tabular}

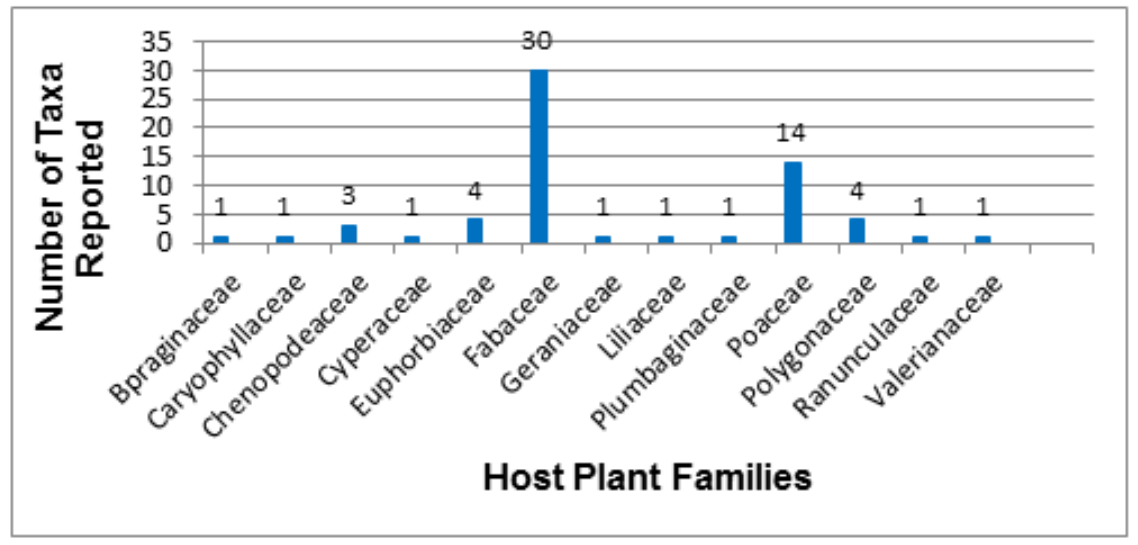

Fig. 2: Graph showing diversity of Uromyces on different host plant families

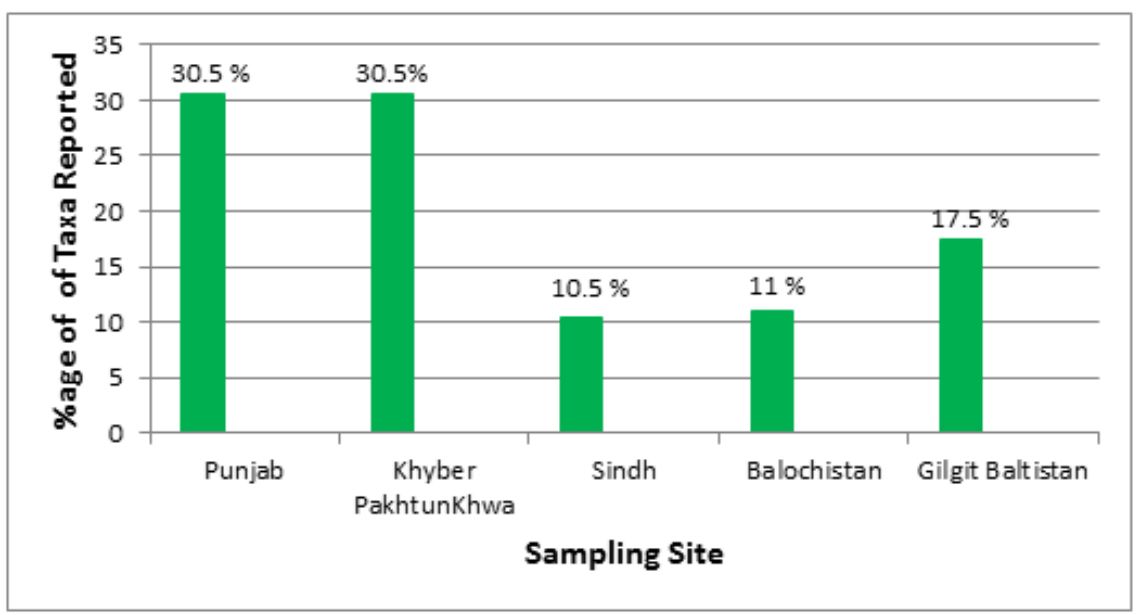

Fig. 3: Graph showing diversity of Uromyces in provinces of Pakistan.

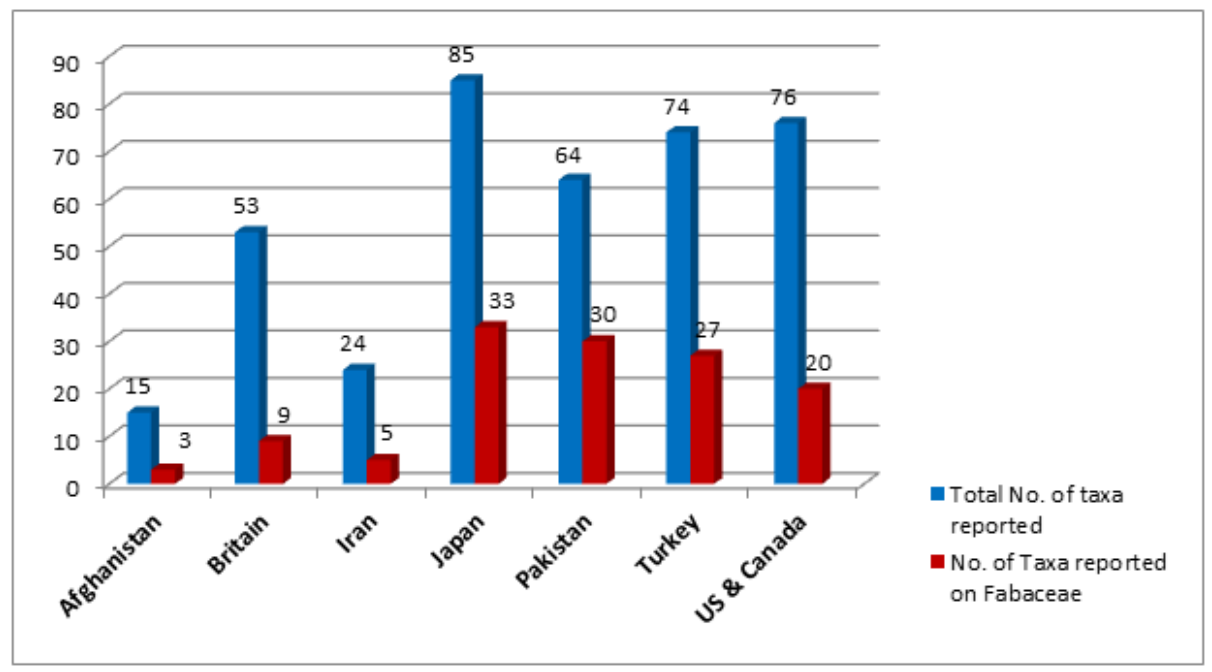

Fig. 4: Graph showing comparative data of host plant families of Uromyces in world. 


\section{Conclusion}

The Uromyces genus is poorly known throughout Pakistan as compared to rest of the world, which is clearly demonstrated by the above described data. An accurate picture of the diversity and distribution of Uromyces throughout the region awaits further surveys.

\section{References}

[1] N. S. Afshan, A. N. Khalid, and A. R. Niazi, "New Records and distribution of rust fungi from Pakistan," Mycotaxon, vol. 105, pp. 257-267, 2008a.

[2] N. S. Afshan, A. N. Khalid, and A. R. Niazi, "New records of Graminicolous Rust Fungi from Pakistan," Pak. J. Bot., vol. 40(3), pp. 1279-1283, 2008b.

[3] N. S. Afshan, A. N. Khalid, and A. R. Niazi, "Some new rust fungi (Uredinales) from Fairy Meadows, Northern Areas, Pakistan," J. Yeast Fungal Res., vol. 3(5), pp. 65-73, 2012.

[4] S. Ahmad, "Uredinales of West Pakistan," Biologia, vol. 2(1), pp. 29-101, 1956a.

[5] S. Ahmad, "Fungi of Pakistan," Biological Society of Pakistan, Lahore, Monograph 1, 1956b, pp. 1-126.

[6] S. Ahmad, "Further contributions to the fungi of Pakistan," II, Biologia, vol. 8, pp. 123-150, 1960.

[7] S. Ahmad, "Contribution to the fungi of Pakistan," IV, Biologia, vol. 10, pp. 1-62, 1964.

[8] S. Ahmad, "Contributions to the fungi of Pakistan," VI, Biologia, vol. 13, pp. 15-42, 1967.

[9] S. Ahmad, "Contributions to the fungi of Pakistan," VII, Biologia, vol. 14, pp. 1-11, 1968.

[10] S. Ahmad, "Fungi of Pakistan," Biological Society of Pakistan, Lahore, Monograph 5, Suppl. 1, pp.110, 1969.

[11] S. Ahmad, "Contribution to the fungi of Pakistan," XVII, Sultania, vol. 2, pp. 17-21, 1976.

[12] S. Ahmad, and M. Arshad, Contributions to the fungi of Pakistan, XII, Biologia, vol. 8, pp. 7-17, 1972.

[13] G. B. Cummins, "Uredinales from the North West Himalayas," Mycologia, vol. 35, pp. 446-458, 1942. http://dx.doi.org/10.2307/3754596

[14] A. Ghaffar, and S. Q. Abbas, “Fungi of Pakistan,” Suppl. II, Pak. J. Bot., vol. 4, pp. 195-208, 1972.

[15] A. Ghaffar, and A. Kafi, "Fungi of Karachi," Pak. J. Sc., vol. 20, pp. 5-10, 1968.

[16] S. Z. Hasnain, A. Khan, and A. J. Zaidi, “Rusts and smuts of Karachi,” Bot. Deptt. Karachi Univ. Mont. Vol. 2, pp. 36. 1959.

[17] A. Ishaq, A. N. Khalid, and N. S. Afshan, "New records of Poaceous rusts from Pakistan," Mycotaxon, vol. 118, pp. 237-244, 2011.

http://dx.doi.org/10.5248/118.237

[18] C. H. Jafri, M.Sc. Thesis, W.P.A.U., Faisalabad, 1968.

[19] I. Jorstad, and S. H. Iqbal, "Uredinales from West Pakistan," Nytt. Mag. Bot, vol. 14, pp. 31-38, 1967.

[20] M. Kakishima, O. Izumi, and Y. Ono, "Rust Fungi (Uredinales) of Pakistan collected in 1991," Cryptogamic Flora of Pakistan, vol. 2, pp. 169-179, 1993.

[21] S. Kaneko, "Parasitic fungi on wood plants from Pakistan," In: Cryptogamic Flora of Pakistan, vol. 2 (EDS. t. Nakaike and S. Malik), pp.149-168. Nat. Sci. Mus., Tokyo, 1993.

[22] A. N. Khalid, S. H. Iqbal, and F. Ahmad, "Rust Flora of Pakistan. Uredinales collected in Salt Range, Pakistan," Sci. Int. (Lahore), vol. 5(2), pp. 211-214, 1993

[23] A. N. Khalid, S. H. Iqbal, and A. Masood, "New records of Uredinales from Pakistan," Sci. Int., vol. 7(4), pp. 531-532, 1995.

[24] A. N. Khalid, and S. H. Iqbal, "Additions to the rust flora of Pakistan," Pak. J. Bot., vol. 28(1), pp. 115-117, 1996.

[25] S. A. Khan, and M. Kamal, "The fungi of South West Pakistan," Pat 1. Pak. J. Sci \& Ind. Res., vol. 11, pp. 61-80, 1968.

[26] S. A. Malik, and M. A. Khan, "Parasitic fungi of North West Frontier Province," Ind. Jour. Agri. Sci., vol. 13, pp. 522-527, 1944.

[27] S. A. Malik, and Virk, "Contribution to the knowledge of parasitic fungi of Quetta-Kalat Region," Biologia, vol. 14, pp. 27-35. 1968. 
[28] S. A. Malik, M. T. Javaid, and M. Ahmad, "Uredinales of Quetta-Kalat region of Pakistan," Biologia, vol. 14, pp. 3746, 1968.

[29] A. Masood, A. N. Khalid, and S. H. Iqbal, "New records of Graminicolous Rust Fungi (Uredinales) from Pakistan," Sci. Int., vol. 7(3), pp. 415-416, 1995.

[30] Y. Ono, "Uredinales collected in the Kaghan Valley, Pakistan," Cryptogamic flora of Pakistan, vol. 1, pp. 217-240, 1992.

[31] Y. Ono, and M. Kakishima, "Uredinales collected in the Swat Valley, Pakistan," Cryptogamic flora of Pakistan, vol. 1, pp. 197-216, 1992.

[32] F. Petrak, "Beitrage Zur Ustilagineen und Urideen Flora on Afghanistan and Pakistan," Sydowia, vol. 2, pp. 278-287, 1966.

[33] M. Saba, A. N. Khalid, and N. S. Afshan, "Species of the genus Uromyces (Basidiomycota: Pucciniales) from Leepa Valley, Azad Jammu and Kashmir (AJ and K), Pakistan,” African Journal of Biotechnology, vol. 10(73), pp. 1648016483, 2011.

[34] M. Saba, A. R. Niazi, and A. N. Khalid, "New records of Uredinales (Basidiomycota) from Azad Jammu and Kashmir and adjacent Northern Areas of Pakistan," Int. J. Agric. Biol., vol. 15, pp. 878-884, 2013.

[35] H. Sydow, and S. Ahmad, "Fungi Punjabensis," Ann. Myc., vol. 37, pp. 439-447, 1939. 